EPJ Web of Conferences 41, 08012 (2013)

DOI: $10.1051 /$ epjconf/20134108012

(C) Owned by the authors, published by EDP Sciences, 2013

\title{
B-side Electron Transfer in Bacterial Photosynthetic Reaction Centers Revealed by a Few-Cycle Pulse Laser
}

\author{
J. $\mathrm{Du}^{1,2}$, T. Kobayashi ${ }^{1,2,3,4}$, K. Watanabe ${ }^{5}$, and H. Tamiaki ${ }^{5}$ \\ ${ }^{1}$ Advanced Ultrafast Laser Research Center, University of Electro-Communications, 1-5-1 \\ Chofugaoka, Chofu, Tokyo 182-8585 Japan \\ 2 JST, CREST, 5 Sanbancho, Chiyoda-ku, Tokyo 102-0075 Japan \\ ${ }^{3}$ Department of Electrophysics, National Chiao-Tung University, 1001 Ta Hsueh Rd., Hsinchu 300 \\ Taiwan \\ ${ }^{4}$ Institute of Laser Engineering, Osaka University, 2-6 Yamada-oka, Suita, Osaka 565-0971 Japan \\ ${ }^{5}$ Department of Pharmacy, Ritsumeikan University, Kusatsu, Shiga 525-8577 Japan
}

\begin{abstract}
B-side electron transfer in wide-type reaction centers from Rhodobacter sphaeroides 2.4.1 was investigated by broadband real-time vibrational spectroscopy. $\mathrm{H}_{\mathrm{B}}{ }^{-}$was found to be generated in less than $50 \mathrm{fs}$ after the excitation of $6.3 \mathrm{fs}$ laser pulse, and its decay lifetime was determined to be $\sim 280 \mathrm{fs}$ and $1.4 \mathrm{ps}$, which is extremely fast compared with the one occurring in A-side. Considering the ultrafast of the generation time of $\mathrm{H}_{\mathrm{B}}{ }^{-}, \mathrm{B}_{\mathrm{B}}$ rather than $\mathrm{P}$ was found to be the initial electron donor to the H. Hence, the initial charge-separated state is determined to be $\mathrm{B}_{\mathrm{B}}{ }^{+} \mathrm{H}_{\mathrm{B}}{ }^{-}$state.
\end{abstract}

\section{Introduction}

The symmetric structure of the reaction center (RC) provides two possible electron-transfer pathways. However, the functional asymmetry has been discovered and only the A-branch is active in charge transfer to form $\mathrm{P}^{+} \mathrm{H}_{\mathrm{A}}{ }^{-}$as the initial charge-separated state when the primary electron donor $(\mathrm{P})$ is excited. The function of the B-branch cofactor is not yet clearly understood and only limited research result has been reported [1-2]. According to these previous studies, the B-branch function is very complicated and it electron transfer pathway is discovered to be dependent on the excitation wavelength, i.e. dependent on whether $\mathrm{P}$ or $\mathrm{B}$ (a monomer bacteriochlorophyll) or $\mathrm{H}$ (a bacteriopheophytin) is excited or to which excited state $\left(\mathrm{Q}_{\mathrm{y}}, \mathrm{Q}_{\mathrm{x}}\right.$, or Soret band) they are excited.

In the present study, we utilize the ultrashort laser pulse at visible range to investigate the kinetic of B-side electron transfer and properties of the initially generated charge pair state. $B_{B}$ was found to act as the initial electron donor rather than $P$. Electron transfer takes place from $B_{B}$ to $H_{B}$ in less than $50 \mathrm{fs}$, and decay components of the charge-separated $\mathrm{B}_{\mathrm{B}}{ }^{+} \mathrm{H}_{\mathrm{B}}{ }^{-}$state are determined to be $\sim 280 \mathrm{fs}$ and $1.4 \mathrm{ps}$, which is extremely fast compared with the one occurring in A-side.

\section{Experimental method and material}

Both pump and probe pulses $(7.2 \mathrm{fs}, 525 \mathrm{~nm}$ to $723 \mathrm{~nm})$ were generated from a noncollinear optical parametric amplifier laser system [3]. All the experiment was performed at a constant temperature

This is an Open Access article distributed under the terms of the Creative Commons Attribution License 2.0, which permits unrestricted use, distribution, and reproduction in any medium, provided the original work is properly cited. 
(293K). RCs from Rhodobacter sphaeroides 2.4.1 were prepared as described previously [4]. The anaerobically grown wide-type (WT) RCs contain spheroidene. RCs were suspended in a buffer solution of $20 \mathrm{mM}$ Tris- $\mathrm{HCl}(\mathrm{PH} 8.0)$ with $0.1 \% \mathrm{~N}$, N-Dimethyldodecylamine-N-oxide.

\section{Results and discussions}
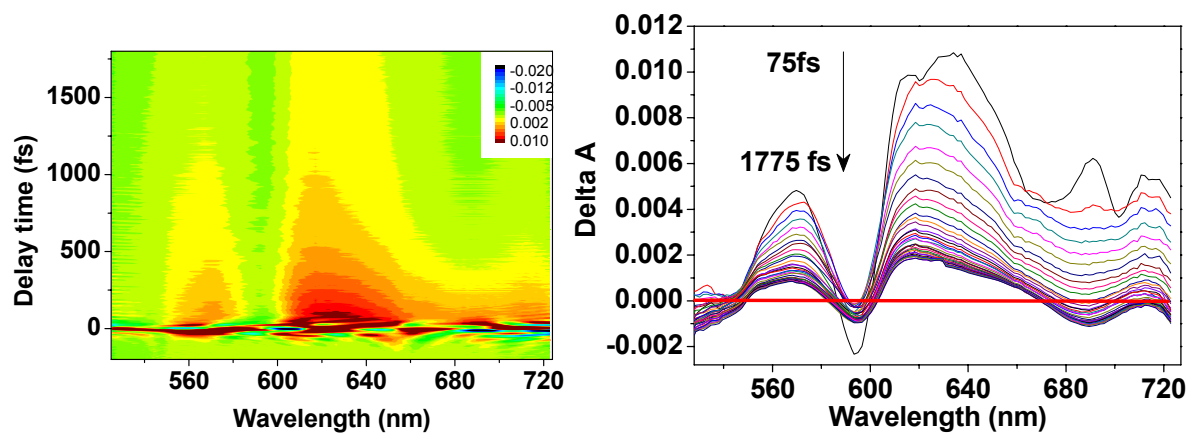

Fig. 1. (a) Two-dimensional (probe photon energy versus probe delay time) pseudo-color display of the time dependence of the absorbance changes $\Delta \mathrm{A}$. (b) The time-resolved difference absorption spectrum probed at delays from 75 to 1775 fs.

Fig. 1a shows the two-dimensionally plotted difference absorption spectra. In Fig. 1b, we plot the probe photon energy dependence of time-resolved spectra from 75 to $1775 \mathrm{fs}$ with an integration width of $50 \mathrm{fs}$. A negative signal at $595 \mathrm{~nm}$ is due to the ground-state bleaching of $\mathrm{P}$ and/or $\mathrm{B}$. There is a strong photon induced absorption (PIA) bands located around $630 \mathrm{~nm}$. According to previous studies, this PIA signal is attributed to formation of the $\mathrm{H}_{B}$ anion [1-2]. Then what is the electron donor for this charge separation? As shown in Fig. $1 \mathrm{~b}, \mathrm{H}_{\mathrm{B}}{ }^{-}$is formed just after the excitation of fewcycle visible laser (shorter than $50 \mathrm{fs}$ ), which is much faster than the one generated under excitation at $390 \mathrm{~nm}$ with laser pulse duration of hundred femtosecond [1]. Thus, the $\mathrm{P}^{+}$seems unlikely to be the initial electron donor, because the formation of $\mathrm{P}^{+}$from $\mathrm{P}^{*}$ usually occurs on the $1 \sim 2$ ps time scale, which is too slow to explain the ultrafast electron transfer to $H_{B}$. Because we excited the $Q_{x}$ state of both $\mathrm{B}$ and $\mathrm{P}$, considering the proximity of $\mathrm{B}_{\mathrm{B}}$ to $\mathrm{H}_{\mathrm{B}}$, the $\mathrm{B}_{\mathrm{B}}$ is much more likely to be the initial electron donor. Thus the corresponding charge-separated state initially formed can be assigned to be $\mathrm{B}_{\mathrm{B}}{ }^{+} \mathrm{H}_{\mathrm{B}}{ }^{-}$, which is similar with the one excited under $390 \mathrm{~nm}$ [1-2]. Femtosecond decay component of $280 \mathrm{fs}$ and picosecond one of $10 \mathrm{ps}$ (maximum delay time is 50ps) have been determined for the $\mathrm{B}_{\mathrm{B}}{ }^{+} \mathrm{H}_{\mathrm{B}}{ }^{-}$state.

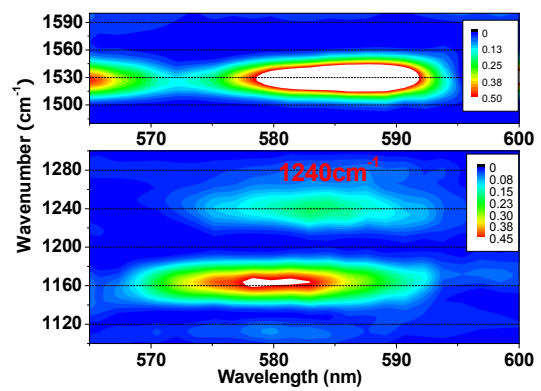

Fig. 2. Two-dimensional contour map of FT amplitude spectra of the pump-probe signal.

The advantage of real-time vibrational spectroscopy is that both the electronic and vibrational dynamics can been observed at the same time under exactly same experimental condition. To gain a 
better understanding of the vibronic coupling mechanisms, the vibrational mode frequencies have been realized by the fast Fourier transform (FFT) analysis of $\triangle \mathrm{A}$, as shown in Fig. 2. The vibrational mode with frequency of $1239 \mathrm{~cm}^{-1}$ can uniquely refer to the cis nature of the $15,15^{\prime}$ ' carbon-carbon double bond as characteristic for spheroidene in the RC [5], which provides a fingerprint by which the carotenoid molecule can be identified. Therefore, it is reasonable to assign the vibrational mode at $1240 \mathrm{~cm}^{-1}$ observed in the range of $570-590 \mathrm{~nm}$ in Fig. 2 due to the carotenoid. Thus, the one located at $1530 \mathrm{~cm}^{-1}$ and $1162 \mathrm{~cm}^{-1}$ can be attributed to $\mathrm{C}=\mathrm{C}$ and $\mathrm{C}-\mathrm{C}$ stretching in the carotenoid, respectively. However spheroidene seems can not be excited directly, the observed signal may be explained by the energy transfer from $B_{B}$ to carotenoid $S_{1}$ state during $\sim 50 \mathrm{fs}$, which has been proved to be an effective path in purple-bacterial photosynthetic core antenna [6].

\section{Conclusion}

Electron at the B-side is found to transfer from $\mathrm{B}_{\mathrm{B}}$ to $\mathrm{H}_{\mathrm{B}}$ in less than $50 \mathrm{fs}$ after the excitation using femtosecond real-time vibrational spectroscopy. Decay components of the initial charge-separated $\mathrm{B}_{\mathrm{B}}{ }^{+} \mathrm{H}_{\mathrm{B}}{ }^{-}$state are determined to be $280 \mathrm{fs}$ and $1.4 \mathrm{ps}$, which is extremely fast in contrast to the processes in A-side. Such short formation and decay time of the electron transfer may arise from the requirement of preventing the photodamage in RCs. Since the A-branch cofactor absorbs the infrared light to undergo electron transfer to convert the light to chemical potential energy, meanwhile, the Bbranch will absorb the light at residual range, i.e., the visible and/or UV light, to avoid the photon damage of these light to the A-branch cofactors and guarantee the successful electron transfer.

\section{Acknowledgments}

This work was supported by the Core Research for Evolutional Science and Technology program of the Japan Science and Technology Agency, National Science Council of the Republic of China, Taiwan (NSC No. 98-2112-M-009-001-MY3), and a grant from the Ministry of Education, Aiming for Top University Program at National Chiao-Tung University. A part of this work was performed under the joint research project of the Institute of Laser Engineering, Osaka University under contract No. A3-01.

\section{References}

1. S. Lin, E. Katilius, A. L. M. Haffa, A. K. W. Taguchi, and N. W. Woodbury, "Blue Light Drives B-Side Electron Transfer in Bacterial Photosynthetic Reaction," Biochem. 40, 13767 (2001).

2. A. L. M. Haffa, S. Lin, J. C. Williams, B. P. Bowen, A. K. W. Taguchi, J. P. Allen, and N. W. Woodbury, "Controlling the Pathway of Photosynthetic Charge Separation in Bacterial Reaction Centers," J. Phys. Chem. B 108, 4 (2004).

3. A. Shirakawa, I. Sakane, and T. Kobayashi, "Pulse-front-matched optical parametric amplification for sub-10-fs pulse generation tunable in the visible and near infrared, " Opt. Lett. 23, 1292 (1998).

4. H. A. Frank, S. S. Taremi, J. R. Knox, "Crystallisation and preliminary X-ray and optical spectroscopic characterisation of the photochemical reaction centre from Rhodobacter sphaeroides strain 2.4.1," J. Mol. Biol. 198, 139 (1987).

5. A. C. Wirtz, M. C. van Hemert, J. Lugtenburg, H. A. Frank, and E.J.J.Groenen, "Two Stereoisomers of Spheroidene in the Rhodobacter sphaeroides R26 Reaction Center: A DFT Analysis of Resonance Raman Spectra," Biophys. J. 93, 981 (2007).

6. D. Kosumi, S. Maruta, T. Horibe, R. Fujii, M. Sugisaki, R. J. Cogdell, and H. Hashimoto, "Ultrafast Energy-Transfer Pathway in a Purple-Bacterial Photosynthetic Core Antenna, as Revealed by Femtosecond Time-Resolved Spectroscopy, "Angew. Chem. Int . Ed. 50, 1097 (2011). 